\section{La Vasectomía como Procedimiento Ambulatorio en el Salvador}

Enrico Henriquez, $\mathrm{MD}^{1}$

Vernon Madrigal, $\mathrm{MD}^{2}$

Lynda Painter Cole, $\mathrm{MA}^{3}$

\section{INTRODUCCION}

La implementación de los programas de planificación familiar en América Latina desde 1960 se ha dirigido principalmente a las mujeres. Se creía que el hombre latino dejaría la planificación familiar a la mujer y que no aceptaría los métodos de control de fecundidad masculina, en particular la vasectomía (3). Una experiencia reciente nos hace dudar de esta hipótesis.

El primer programa público de vasectomía en América Latina comenzó en Bogotá en febrero de 1970; desde entonces, se han iniciado programas en Costa Rica (1971), El Salvador (1972) y Guatemala (1973) (3). La experiencia colombiana ha sido tan exitosa que fue necesario aumentar el número de centros que ofrecían el procedimiento. La vasectomía probablemente no ha sido aceptada en América Latina simplemente porque no se ha ofrecido.

La Asociación Demográfica Salvadoreña (ADS), una clínica privada de planificación familiar en San Salvador, El Salvador, ofrece la vasectomía como parte integral de sus servicios. Las operaciones son efectuadas por gíneco-obstétras y no por urólogos. Este trabajo relata la experiencia inicial de los servicios de vasectomía en la ADS, con el objeto de determinar la posibilidad de ofrecer este método en otras clínicas de planificación familiar.

\footnotetext{
1 Cirujano

Asociación Demográfica Salvadoreña

San Salvador, El Salvador

2Hasta Junio de 1977, Direct or Médico Asociación Demográfica Salvadoreña

San Salvador, El Salvador

3Senior Research Analyst

International Fertility Research Program Borth Carolina, USA
}

Este trabajo fue realizado bajo los auspicios del International Fertility Research Program y la Oficina de Población de la Agencia Internacional de Desarrollo de los Estados Unidos (AID/phaC-1172).

\section{METODO}

Desde febrero 1974 hasta noviembre 1975,200 hombres solicitaron vasectomías en la ADS. Datos sociodemográficos y médicos fueron registrados en formularios por el personal del centro. Todos los pacientes debían regresar a visitas de control a los ocho días y a los seis meses de la esterilización. Se obtuvo también por lo menos un espermiograma después del procedimiento.

\section{RESULTADOS}

\section{Características de los pacientes}

La mayor parte de los hombres eran casados $(92.5 \%)$, católicos $(85.0 \%)$ y vivían en el área urbana de San Salvador (81.5\%).

El Cuadro I muestra las características sociodemográficas de los pacientes casados y de sus esposas. La edad promedio de los vasectomizados era 34.3 años y el nivel de educación promedio era 7.1 años. La mayoría eran trabajadores semícalificados $(35.5 \%)$ ú operarios $(31.0 \%)$.

Datos con respecto a las esposas muestran que éstas eran más jóvenes y con menos educación escolar que los hombres. La edad promedio de las esposas era 28.7 años y habían completado un promedio de 5.7 años de educación formal. La mayor parte de las esposas $(71.5 \%)$ trabajaban en su hogar.

Se compararon datos sociodemográficos de parejas que habían elegido la esterilización femenina (725 durante este período de tiempo) y aquellas que habían elegido la vasectomía. Las mujeres resultaron similares en cuanto a edad y empleo. La edad promedio de las mujeres esterilizadas era 29.2 años y el $66.1 \%$ trabajaban en su hogar. Las parejas que eligieron vasectomía como un método de anticoncepción permanente tenían un nivel educacional más alto. Las mujeres esterilizadas tenían un promedio de 3.9 años de educación, comparado con 5.7 años para las esposas de los hombres vasectomizados. Los hombres vasectomizados tenían un nivel educacional promedio de 7.1 años, comparado con $\mathbf{5 . 2}$ años para los esposos de las mujeres esterilizadas. 
Historia Médica, Reproductiva y Anticonceptiva

Los datos sobre historia reproductiva fueron recolectados solamente de la mitad de los hombres que participaron en este estudio. Estos hombres reportaron tener un promedio de 4.6 hijos, del cual un promedio de 4.2 hijas vivían al momento de la vasectomía (Cuadro I). Las mujeres esterilizadas en la ADS tenían una paridad pro- medio de 4.2.

Casi el diez por ciento de los hombres se vasectomizaron cuando tenían sólo uno o dos hijos vivos. Debemos notar que 5 de los casos tenían sólo hijas mujeres, de 3 los casos tenían sólo varones y los 11 restantes tenían un hijo de cada sexo. Esto es totalmente contrario a la idea común que los hombres latinos insistirían en tener un hijo antes de considerar la esterilización.

\section{CARACTERISTICAS SOCIODEMOGRAFICAS SELECCIONADAS DE 200 HOMBRES VASECTOMIZADOS EN LA UNIVERSIDAD DE EL SALVADOR DESDE FEBRERO 1974 HASTA NOVIEMBRE 1975 Y SUS ESPOSAS}

Edad

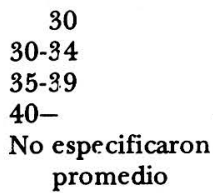

Educación

Niriguna

1-6

$7-12$

13-

Promedio

\section{Ocupación}

Profesional

Gerente/propietario/ dueño de tierra

Oficinista

Artesano

Operador

Jornalero/labores

agrícolas

Otro

Cesante

No especificaron

\section{Estado Civil}

Casado, una esposa

Casado, más de una vez

Casado ant eriormente

Hijos

1-2

$3-4$

$5-6$

$7-$

Pacientes

(N-200)

27.0

31.0

18.5

23.0

\section{Esposas}

(N-183)

$\begin{array}{cr}112 & 61.2 \\ 39 & 21.3 \\ 20 & 10.9 \\ 12 & 6.6\end{array}$

28.7

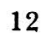

102

68

18

26

17

5

8

71

62

3

3

5

183

15

16

41

20

17

100

\section{0}

51.0

34.0

9.0

7.1

17
114
48
4

9.3

62.3

26.2

2.2

5.7

8.2

0.5

1.6

2.2

2.7

4.9

4.9

71.5

3.3
No especificaron

91.5

1.0

7.5

Promedio 
El cuarenta y tres por ciento de los hombres reportaron que ni ellos ni sus esposas habían usado un método anticonceptivo durante los tres meses previos a la esterilización; el 26.5\% de las parejas usaron la vía oral, el $14.5 \%$ usaron condones y el $7.5 \%$ usaron dispositivos intrauterinos. Dos hombres reportaron que sus esposas habían sido esterilizadas. Sin embargo, sólo el $2.3 \%$ de las mujeres esterilizadas en la ADS reportaron no usar anticonceptivo anteriormente a la esterilización; el $61 \%$ usaron la vía oral y el $35.0 \%$ usaron los DIUS.

\section{Motivación}

La mayor parte de estos hombres fueron referidos a la ADS por otros hombres que habían sido vasectomizados $(37.0 \%)$ o por el personal de planificación familiar (19.5\%). Solamente el $13.0 \%$ reportó que su esposa o él mismo había sido la fuente principal de referencia. Cuando le preguntaron a la mitad de los hombres porqué habían seleccionado la vasectomía en vez de otro método de planificación familiar, el $40.0 \%$ contestó que los otros métodos no eran seguros y el $31.0 \%$ tenían dudas sobre los efectos colaterales de los otros métodos.

Se encontraron condiciones médicas preexistentes en el $17.5 \%$ de los pacientes. La más frecuente de éstas fue dolor del escroto y los testículos $(5.0 \%)$.

\section{Procedimientos Clínicos}

Cada hombre que se interesó en una vasectomía fue entrevistado por una trabajadora social. Si el hombre eligió la operación, se le dió cita para regresar a la clínica. No se le dió instrucciones preoperativas a ninguno de los hombres.

Antes de que el paciente fuera admitido a la sala de operación, se le afeitó el escroto y se le lavó con jabón y agua. Después que el paciente entró en la sala de operación, se preparó el área del escroto usando una solución antiséptica. No se uso ningún medicamento preanestésico.

En el $88.0 \%$ de los procedimientos, se infiltró 10 cc de lidocaína al $1 \%$ a cada lado en la parte superior del escroto a 2 $\mathrm{cm}$ del anillo inguinal. El conducto deferente fue identificado, aislado y tomado con una pinza de Allis. Una incisión verti- cal de $1 \mathrm{~cm}$ fue efectuada, el conducto deferente fue aislado y disecado con una pinza curva de Murphy. Se efectuó una ligadura sencilla usando catgut crómico " 00 ” y se efectuó una ligadura doble en el extremo proximal, doblando el conducto sobre si mismo. El segmento entre las dos ligaduras fue seccionado. Se confirmó la hemostasis y se cerró la incisión con dos puntos de crómico " 00 ". La misma técnica fue usada en el $22.0 \%$ de los procedimientos restantes, con excepción de que éstos se efectuaron a través de una incisión media. El paciente pasó a la sala de recuperación donde permaneció aproximadamente dos horas. La operación fué efectuada en un promedio de 16.3 minutos.

Se le instruyó al paciente que esperara al menos 24 horas antes de resumir sus actividades normales. Se le aconsejó a cada paciente que usara otros anticonceptivos hasta que la esterilidad se confirmara con un espermiograma negativo, el cual se efectuaría después de por lo menos quince eyaculados, o en la visita de control a los tres meses del procedimiento.

\section{Complicaciones y Quejas}

No ocurrieron complicaciones durante o inmediatamente después de la cirugía. Se le preguntó a la mitad de los hombres si habían sentido algún dolor y el $14.0 \%$ reportó dolor leve.

Durante la visita de control a la semana del procedimiento, se registraron complicaciones en el $10.3 \%$ de los pacientes que regresaron para el seguimiento y el $15.2 \%$ registraron quejas (Cuadro II). La complicación registrada con más frecuencia fué infección de la herida $(5.4 \%)$ pero ninguna de las infecciones fue lo suficientemente severa como para necesitar tratamiento. E1 $12.5 \%$ de los hombres reportó dolor leve, el $1.1 \%$ dolor moderado y el $1.6 \%$ dolor severo.

Gran parte de los hombres regresaron a la visita de seguimiento de cuatro a seis semanas después de la cirugía. De éstos, el $1.7 \%$ reportó complicaciones y el $2.8 \%$ reportó quejas (Cuadro II).

\section{Fallas y Fallas Potenciales}

No se habían reportado embarazos al momento de análisis, más de un año des- 


\section{COMPI.ICACIONES Y QUEJAS DE 200 HOMBRES VASECTOMIZADOS}

EN LA UNIVERSIDAD DE EL SALVADOR

DESDE FEBRERO 1974 HASTA NOVIEMBRE 1975

Complicaciones al Seguimiento Precoz

(de una semana) $(\mathrm{N}-184)$

$\begin{array}{cc}\text { No. } & \text { \%o } \\ 10 & 5.4 \\ 3 & 1.6 \\ 1 & 0.5 \\ 2 & 1.1 \\ 1 & 0.5 \\ 1 & 0.5 \\ 1 & 0.5 \\ 19 & \\ & 10.3\end{array}$

Herida séptica

Hematoma escortal

Hidrocele

Granuloma

Infección local de la piel

Herpes de Zoster (glándula)

Sangrado a las primeras relaciones sexuales

Total de hombres con complicaciones

al seguimiento precoz

Quejas al Seguimiento Precoz (N-184)

Dolor leve

Dolor moderado

Dolor severo

Total de hombres con quejas al seguimiento precoz

Comp'icaciones al Seguimiento Tardío

(de uno a seis meses) $(\mathrm{N}-180)$

Granuloma

Dolor en los testículos

Dolor lumbar agudo (probablemente

relcionado al trabajo)

Total de hombres con complicaciones al seguimiento tardío

Quejas al Seguimiento Tardío (N-180)

Diminución de actividad sexual

Diminución de ejaculado

Impotencia (tratada antes de la cirugía)

Total de hombres con quejas

pués de efectuarse el último procedimiento.

A 136 hombres que regresaron al seguimiento de uno a seis meses se les efectuó un espermiograma. Para este análisis los hombres que tuvieron espermiogramas positivos fueron considerados como fallas potenciales de método. Cuatro $(2.9 \%)$ de los hombres tuvieron espermiogramas positivos (con evidencia de espermatozoides móviles o no). Dos no regresaron para el segundo espermiograma y los otros dos tuvieron espermiogramas positivos seis meses después del procedimiento. El resultado no es concluyente ya que el seguimiento al año puede haber resultado negativo o el conteo de espermatozoides puede haber sido demasiado bajo para impregnar.

\section{DISCUSION}

Este análisis demuestra que la vasectomía es un método permanente de anticoncepción masculina seguro, relativamente efectivo y aceptable para muchas de las parejas salvadoreñas que acudieron a la ADS para esterilización. La tasa de complicación resultó baja, a excepción de la tasa de infección leve de la herida notada al seguimiento de una semana. Aunque esta infección puede ser una complicación de cualquier operación, se deben hacer esfuerzos para disminuir dicha tasa. El dolor reportado por los 
pacientes, en la mayoría de los casos, era leve y se alivió con analgésicos. La tasa de fallas potenciales fue más baja que la reportada en otra investigación en América Latina (2).

Aunque el nivel educacional de las parejas que eligieron vasectomía era más alto que el de aquellas que seleccionaron la esterilización femenina, un porcentaje mayor no había utilizado anticoncepción antes de la esterilización. Presser (4) encontró diferencias de educación en un estudio de parejas puertorriqueñas, pero aquellos datos no indicaron ninguna diferencia en el uso de anticonceptivos.

Más de un tercio de los hombres se enteraron del programa de vasectomía en la ADS a través de otros hombres vasectomizados. Aquellos previamente vasectomizados que recomendaron la ADS al parecer estaban satisfechos con su experiencia.

Cuarenta y cinco campesinos que habían sido vasectomizados en la ADS desde 1972 participaron en una discusión en grupo (1). La opinión y el ejemplo de los líderes sindicales, además de la presión económica que causa el número de hijos sobre una familia con recursos limitados, influenciaron a los hombres a obtener la vasectomía. Los hombres expresaron satisfacción con el procedimiento, diciendo que sus relaciones sexuales habían mejorado y eran más placenteras ahora que no había temor de otros embarazos. Aunque algunos de los hombres tuvieron dolor postoperatorio, en la mayor parte de las operaciones no hubieron ni complicaciones ni quejas.

La vasectomía recién se ha comenzado a ofrecer en América Latina. El aumento en su popularidad en aquellos países donde se ofrece el procedimiento está disipando la idea que la vasectomía no puede ser aceptada en países con tradición de dominación masculina.

\section{RESUMEN}

La experiencia de 200 hombres vasectomizados en la Asociación Demográfica Salvadoreña (ADS), una clínica privada de planificación familiar, muestra que la vasectomía es un método seguro y efectivo de anticoncepción permanente, aceptado por muchas parejas salvadoreñas. Las parejas que solicitaron esterilización masculina en la ADS tenían más años de educación escolar que aquellas que solicitaron esterilización femenina, pero por lo general no habían usado otros métodos anticonceptivos antes de la esterilización. Más de un tercio de los pacientes se enteraron del programa de vasectomía a través de otros hombres previamente esterilizados. La vasectomía probablemenye no ha recibido una aceptación más amplia en la América Latina por no haberse ofrecido el procedimiento en mayor escala.

\section{SUMMARY}

The experience of 200 vasectomized men in the Salvadorean Demographic Association (Asociación Demográfica Salvadoreña ADS), a private family planning clinic, demonstrates that vasectomie are a safe and effective method for permanent birth control accepted by many Salvadorean couples. The couples resting masculine sterilization at ADS had more school education than those requesting femenine sterilization, but, in general, had not used other birth control methods before sterilization. More than one third of the patients became aware of the vasectomy program through other men who had undergone the sterilization. Vasectomies have probably not acquired a wider acceptance in Latin America because the procedure has not been offered in a larger scale.

\section{BIBLIOGRAFIA}

1. CASTANEDA RUGAMAS, R.: Cifras y Testimonios de Vasectomizados de la Unión Comunal Salvadoreña (U.C.A.), Asociación Demográfica Salvadoreña, San Salvador, E1 Salvador, Enero, 1976.

2. GALICH, L. F., ESCOBEDO, G. ELKINGTON, J. S. H., McCANN, M. F.: Male Sterilization in Guatemala. Presentado en el Congreso Anual del International Family Planning Research Association, Beverly Hills, California, Septiembre 29-Octubre 2, 1976.

3. MADRIGAL, V., EDELMAN, D. A., GOLDSMITH, A.: Male Sterilization in El Salvador: A Preliminary Report. J. Reprod Med 14: $167,1975$.

4. PRESSER, H. B.: Male versus Female Sterilization: Couple Selectivity and the Role of the Physician. En Sciarra, J. J., Markland, C. y Speidel, J. J., Control of Male Fertility, Program for Applied Research on Fertility Regulation, University of Minnesota, 1975. 